\title{
Thiamine Limited Steady State Growth of the Yeast Cryptococcus albidus
}

\author{
By D. K. BUTTON \\ Institute of Marine Science, University of Alaska \\ College, Alaska 9970 I, U.S.A.
}

(Accepted for publication 8 May I969)

\begin{abstract}
SUMMARY
The concentration/growth rate relationship of thiamine-requiring Cryptococcus albidus was examined. Data suggest a diffusion-limited mechanism characterized by an apparent Michaelis constant for growth of $4 \cdot 7 \times 10^{-13} \mathrm{M}$. This relationship was obtained from continuous culture at steady state and tested by a non-steady state procedure and in batch growth. It was concluded that most natural water systems have sufficient thiamine to support some thiamine-requiring micro-organisms at above rate limiting concentrations.
\end{abstract}

\section{INTRODUCTION}

While the concentration controlled rate of thiamine incorporation has been studied in yeast (Ziro, 1955) and bacteria (Neujahr, 1966), the concentrations of vitamins over which vitamin-limited growth rates of heterotrophs are controlled remain in question. Vitamins are present in most natural, terrestrial and marine systems, and their distribution in sea water has been measured (Holm-Hansen, Strickland \& Williams, 1966; Neujahr, 1966; Natarajan \& Dugdale, 1966). One result of the rather ubiquitous distribution of vitamins in measurable quantities is that most species of micro-organisms absorb part of their vitamin-coenzyme complement from the surroundings and consequently develop vitamin requirements. The facility with which micro-organisms incorporate required vitamins becomes one factor in nutritional chemistry upon which the resulting ecosystem depends. The following paper describes steady state growth rates of a marine yeast, Cryptococcus albidus, with respect to thiamine concentration.

\section{METHODS}

Organism and cultivation. Cryptococcus albidus, the thiamine bioassay organism of Natarajan \& Dugdale (I966), was selected because of its thiamine requirement, marine origin and amenability to continuous culture. Growth was in a $500 \mathrm{ml}$. reactor vessel supplied with a glucose + mineral salts medium at a controlled rate from a 20-1. carboy at $25^{\circ}$ as described elsewhere (Button, 1969a). Although oscillations in the organism population density occurred under some nutritional conditions, such as manganese deficiency (D. K. Button, unpublished data), the thiamine-limited steady state was smooth and constant.

Thiamine-free glucose was prepared by irradiating the required amount of recrystallized glucose in a small quartz tube for $\mathrm{I}$ hr with an ultraviolet lamp. The half-life of thiamine is of the order of minutes under these conditions (Button, 1968). The feed 
carboy and mineral salts were subjected to several hours of irradiation. Purified glucose was added to $100 \mathrm{mg}$./1., and the medium immediately autoclaved.

Limiting substrate. A stock solution of thiamine, which is stable in acid solution (Koser, 1968), was autoclaved for $5 \mathrm{~min}$. at $\mathrm{pH} \mathrm{2,} \mathrm{I}_{2} \mathrm{I}^{\circ}$, and kept in $\mathrm{IO}^{-7} \mathrm{M}$ solution. Sterility tests were occasionally made by spreading a sample on nutrient agar plates. Bioassay showed that freshly prepared thiamine solution had the same activity as 2-year-old stock. The stock solution was diluted as required and added directly to the feed carboy.

Population measurements. Populations were determined by standard plate count techniques and by electronic counting and sizing (Coulter Counter Model B, Hialeah, Florida). Normally Io to 15 volumes were allowed to pass through the reactor in the process of establishing the steady state values reported.

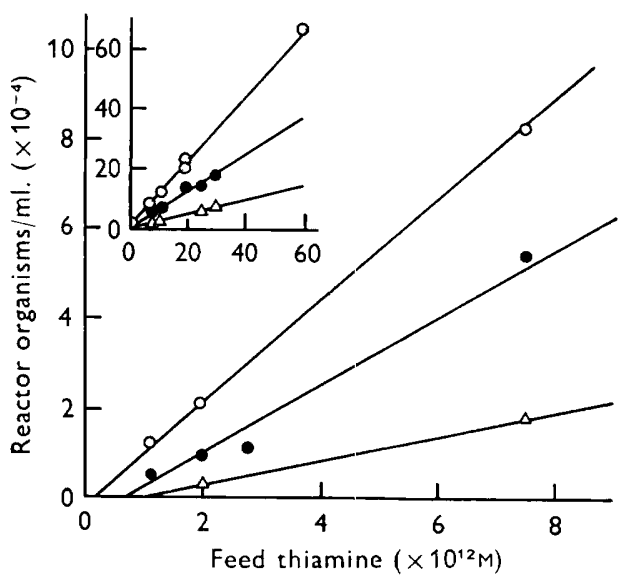

Fig. I

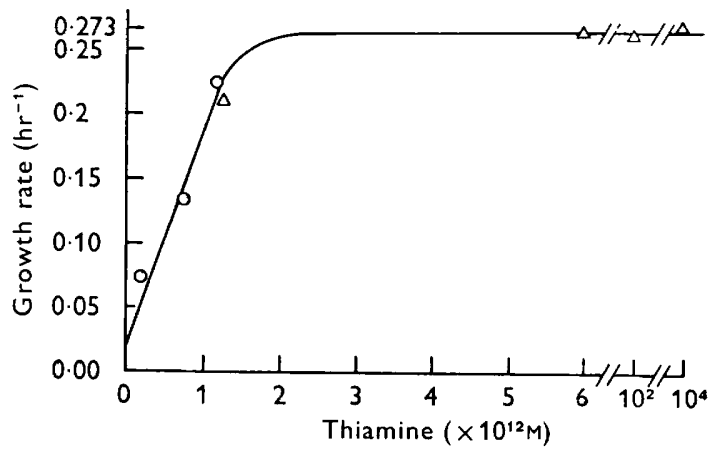

Fig. 2

Fig. I. Cryptococcus albidus. Steady state thiamine limited reactor populations at dilution rates equivalent to $30 \%, O ; 50 \%, 8$; and $80 \%, \triangle$, of a dilution rate equivalent to the maximum growth rate of $0.273 \mathrm{hr}^{-1}$. The inset extends the concentration range of the data.

Fig. 2. Relationship between thiamine concentration and growth velocity of Cryptococcus albidus. Circles represent intercepts from Fig. I. Triangles are taken from washout data according to equation (3).

\section{RESULTS}

Thiamine concentrations controlling growth velocities in their mid-velocity range were determined by the zero population extrapolation point by using a plot of input thiamine versus steady state population (Button \& Garver, I966). Figure I shows the data used for extrapolation at growth rates corresponding to 30,50 , and $80 \%$ of $\mu_{\max }$, the maximum growth rate of Cryptococcus albidus under conditions specified. The extrapolated values, equivalent to extracellular thiamine concentrations, were $\mathrm{I} \times 1 \mathrm{IO}^{-13}, 6 \times \mathrm{IO}^{-13}$ and $8 \times \mathrm{IO}^{-13} \mathrm{M}$, respectively. A complete set of data over a large concentration range is shown in the inset. Intercepts of the population data on the substrate axis are replotted in Fig. 2.

This determination of extracellular thiamine concentrations at various growth rates was tested in two ways. The first method was designed to yield concentration informa- 
tion at velocities approaching the maximum growth rate. After an initial steady state had been reached, the reactor population was adjusted to some low value with respect to thiamine concentration by an increase in feed rate for a suitable period. After obtaining the desired population density, the feed rate was readjusted to a value near the expected growth rate, and a period of several hours was allowed for growth rate stabilization. Then the rate of reactor population change was measured and the growth rate calculated from the usual equation describing population growth in a perfectly mixed continuous flow reactor diluted by sterile feed:

$$
d x / d t=\mu X-r X,
$$

where $\mu$ is the specific growth rate and $r$ the feed rate into the reactor divided by its volume, each having units of time ${ }^{-1}$. Integrating between initial and final organism concentrations, $X_{0}$ and $X$, over a time interval, $t$, yields the exponential relationship

$$
X=X_{0} e^{(\mu-r) t},
$$

where $\mu-r$ is the slope of a line describing $\ln X$ with respect to time. If the dilution rate is set approximately equal to the expected growth rate then $\mu$ can be determined to the same order of accuracy as the feed rate, since the reactor is filled and its volume fixed. The pump-controlled feed rate variation was less than $\mathrm{I} \%$. Extracellular thiamine concentration is simply the difference between the input thiamine concentration, $S_{0}$, and the organism concentration, $X$, times the appropriate organism yield, $\gamma$, as shown in equation (3).

$$
S=S_{0}-X / \gamma
$$

Table I relates organism yield to thiamine used. These data were obtained from electronic counter size distributions and yields calculated from the slopes of the lines from Fig. I.

Table I. Cryptococcus albidus: influence of specific growth rate (dilution rate) on organism size and growth yield from thiamine

$\begin{array}{cccc}\begin{array}{c}\text { Dilution rate } \\ \left(\% \mu_{\max }\right)\end{array} & \begin{array}{c}\text { Organism } \\ \text { volume (average) }\end{array} & \begin{array}{c}\text { Thiamine } \\ \text { molecules/organism }\end{array} & \begin{array}{c}\text { g. organism/ } \\ \text { g. thiamine }\end{array} \\ \text { I 5 } & - & 1.7 \times 10^{4} & - \\ 30 & 38 \mu \mathrm{m}^{3} & 5.3 \times 10^{4} & 29.0 \times 10^{5} \\ 50 & 40 \mu \mathrm{m}^{3} & 9.0 \times 10^{4} & 18 \cdot 0 \times 10^{5} \\ 80 & 70 \mu \mathrm{m}^{3} & 24.0 \times 10^{4} & 1 \cdot 2 \times 10^{5}\end{array}$

Growth rate data obtained by this method and corrected for thiamine used according to Table $I$ are shown as triangles in Fig. 2. The growth rate data at high thiamine concentrations determined from batch growth curves were identical with those shown in Fig. 2 above $6 \times \mathrm{IO}^{-12} \mathrm{M}$. Concentrations of thiamine as high as $\mathrm{I} \times \mathrm{IO}^{-6} \mathrm{M}$ showed no inhibition. An example of a non-steady state experiment at lower thiamine concentrations is shown in Fig. 3. This experiment yielded data in the region of the Michaelis constant and is plotted at $0.2 \mathrm{I} \mathrm{hr} \mathrm{h}^{-1}$ in Fig. 2. Although this type of experiment was designed for precision at higher growth velocities, the agreement between these data and the extrapolation data shown as circles in Fig. 2 is reasonably good. The data reported in Fig. 2, when computed fit to a hyperbola, yielded an apparent Michaelis constant for growth, $K_{s}$, of $4.7 \times 10^{-13} \mathrm{M}$ with a variance of $0.4 \times 10^{-13} \mathrm{M}$ and a maximum growth velocity of $0.273 \mathrm{hr}^{-1}$ with a variance of $0.012 \mathrm{hr}^{-1}$.

Steady state nutrient limited cultures normally respond to a change in limiting 
nutrient concentration by a major change in division rate. This phenomenon was used to test the reported Michaelis constant by a second method. A small number of organisms from a steady state thiamine limited culture growing at half-maximum rate was introduced into $100 \mathrm{ml}$. sterile quartz tubes containing various concentrations of thiamine in a medium which had been purified by irradiation. Care was taken to effect a rapid transfer of organisms from steady state and to avoid temperature fluctuation. The cultures were shaken in an incubator at $25^{\circ}$ and the rate of population change measured as shown in Fig. 4. The growth rate of the inoculum in continuous culture was $0.132 \mathrm{hr}^{-1}$. Of the thiamine concentrations used, that which effected the least change in growth rate after rapid transfer to the batch system was $\mathrm{I} \times \mathrm{IO}^{-13} \mathrm{M}$.

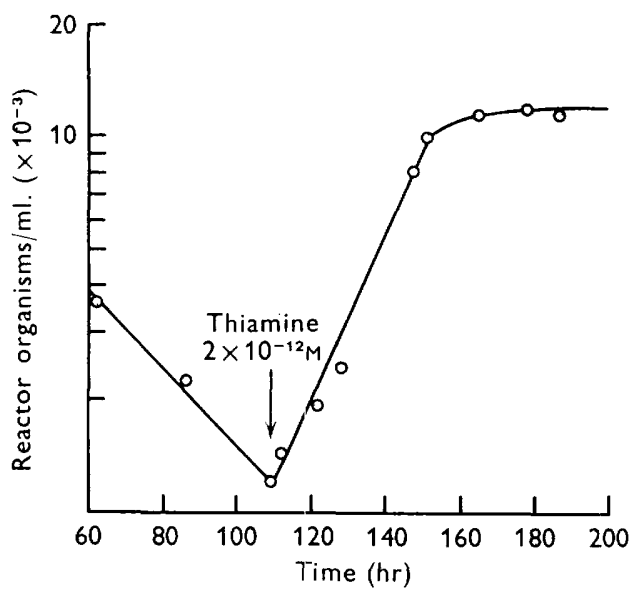

Fig. 3

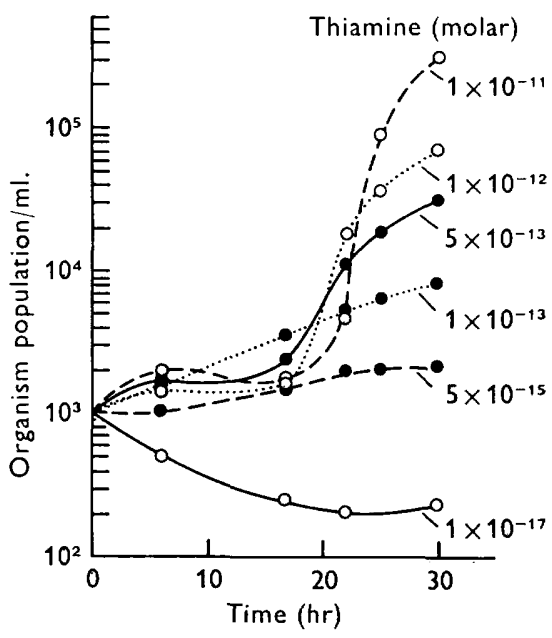

Fig. 4

Fig. 3. Cryptococcus albidus. Reactor population, dilution rate $0 \cdot 132 \mathrm{hr}^{-1}$. The arrow indicates a change of feed thiamine level from $5 \times 10^{-13} \mathrm{M}$ to $2.5 \times 10^{-12} \mathrm{M}$.

Fig. 4. Batch growth curves of Cryptococcus albidus at initial thiamine concentrations indicated. An inoculum of 1000 organisms $/ \mathrm{ml}$. was obtained from a steady state continuous culture diluted at a rate of $0.132 \mathrm{hr}^{-1}$.

The resulting growth rate with this concentration was $0.099 \mathrm{hr}^{-1}$, midway between the inoculum growth rate from continuous culture and the value $0.04 \mathrm{I} \mathrm{hr}^{-1}$ predicted by the Michaelis-Menten equation using $K_{s}$ reported above. These batch growth rates are thus compatible with the Michaelis constant determined at steady state from continuous culture data. Higher concentrations consistently produced non-steady state behaviour as shown by the non-linearity of the exponential curve in Fig. 2. After the lag induced by the increase in limiting substrate the growth sustained a rate greater than $\mu_{\max }$ for a few divisions. This new division rate also follows a saturation curve with respect to initial thiamine concentration. The concentration producing the half maximal rate for this phenomenon is $\mathrm{I} \times \mathrm{IO}^{-12} \mathrm{M}$.

Effects of temperature, $\mathrm{pH}$ value and mixing energy on the growth velocity/concentration relationship were examined by arranging a thiamine limited steady state population in continuous culture at sufficiently low population density so that a significant portion of the thiamine supplied by the feed remained in the extracellular 
phase. Changes in the concentration/velocity relationship are then refiected by changes in the steady state organism population. When the feed of a continuous culture system at $\mathrm{pH}_{4}$ was titrated to $\mathrm{pH} 6$ while in operation at half maximum growth rate, no change in the extinction value 0.004 the population density was observed.

The effect of temperature on the population is shown in Table 2. The growth rate was set at $80 \%$ of $\mu_{\max }$ at the lower temperature so that the population over the whole range of temperatures could be observed at the same specific growth rate, $0.218 \mathrm{hr}^{-1}$. The decrease in population density at the highest temperature is accounted for by a shift of the size distribution toward larger organisms at the higher temperature. These data showed no increase in mass of organism derived from a given thiamine concentration with increasing temperature.

Table 2. Cryptococcus albidus: influence of temperature on number of organisms $/ \mathrm{ml}$. (growth rate $=0.80 \mu_{\max }$ )

$\begin{array}{cc}\text { Temperature } & \text { Organisms } / \mathrm{ml} . \\ 17 \cdot 0^{\circ} & 24,400 \\ 25 \cdot 0^{\circ} & 18,600 \\ 29 \cdot 5^{\circ} & 8,488\end{array}$

Table 3. Cryptococcus albidus: effect of power input (mixing efficiency) on organism concentration with growth rate $=0.80 \mu_{\max }$

$\begin{array}{cc}\text { Watts per l. } & \text { Organisms } / \mathrm{ml} . \\ \text { O.I } & 9,520 \\ \text { I.4 } & \text { I } 5,120\end{array}$

A change in reactor stirring rate did, however, affect the total population. Table 3 shows the population under identical concentrations of thiamine limited steady state growth, but at different stirring rates of 856 and $85 \mathrm{rev} . / \mathrm{min}$. Stirring was provided by a I-in. $(2.54 \mathrm{~cm}$.) teflon-coated magnet. Growth rate was half maximum and agitation was changed by varying the speed of the magnetic stirrer as described by Borkowski \& Johnson (1967). A significant difference in population density was consistently observed with changes in agitation power input.

\section{DISCUSSION}

The value of $4.7 \times 10^{-13} \mathrm{M}$ was established as the mid-range for thiamine concentration controlled growth rate of Cryptococcus albidus. This value was confirmed by two independent methods, since the concentrations involved were much lower than those usually measured when examining growth kinetics; the results were compatible with the value reported. Concentration data in the region of $\mu_{\max }$ were fairly precise and suggested deviation from this hyperbolic relationship. Although the data in the region of the Michaelis constant were less precise, the data in this region are best fit with a straight line as shown in Fig. 2 and are suggestive of a diffusion limited mechanism. Deviation from the origin is within the experimental error reported; however, this deviation can also reflect the degree of contamination by background thiamine. The concept of a diffusion limited mechanism was supported by the fact that changes in 
temperature or $\mathrm{pH}$ value did not affect the velocity of the rate limiting step. More energetic mixing did however increase the efficiency of thiamine absorption. As reported elsewhere (Button, 1969b), the effective concentration of thiamine in the bulk of the medium is approximately doubled by mixing in this diffusion limited system over the normal growth rate range of the yeast. Diffusion to and across the cell wall appears to be sufficient at the concentrations reported to provide the thiamine flux observed. Borkowski \& Johnson (1967) provided a useful derivation and discussion concerning oxygen supply which can be applied to this calculation.

Short-term division rates were significantly faster than the long-term value of $\mu_{\max }$ when a thiamine limited culture was supplied with excess thiamine. This suggests the accumulation during thiamine limited growth of some component whose supply is normally the rate limiting step. The Michaelis constant for growth is directly related to $\mu_{\max }$, hence the short-term high growth rate is associated with a slightly larger Michaelis constant.

Most organic nutrients are actively transported into the organism (Müller, 1967). If there is an initial common carrier (Hengstenberg, Egan \& Morse, 1967) one can calculate a lower limit for the amount of carrier required to give a given flux at the reported concentrations of substrate (Amdur \& Hammes, 1966). By using the upper limit for bimolecular reactions in liquids of $10^{9} \mathrm{M}^{-1} \mathrm{sec}^{-1}$ and a carrier molecular weight of 30,000 the observed flux would require that the organism be composed of only $0.01 \%$ of this carrier. A carrier concentration of around I \% would bring the reaction rate down to within the range of many enzymes. Thus the Michaelis constant of $4.7 \times 10^{-13} \mathrm{M}$ is reasonable for either a diffusion or an active transport mechanism, even though it is a lower concentration than usually considered in nutritional chemistry.

Although other workers (Jannasch, 1967; R. D. Hamilton, personal communication) have reported that the yield constants in their systems were not constant, we did not find that to be the case, as shown by the linearity of the data shown in Fig. I. The yield constant does change with growth rate because larger amounts of the coenzyme are required to catalyse the higher metabolic rates at the faster growth rates, but it is constant at a given growth rate.

Concentrations of thiamine in the oceans are in the range of $10^{-10}$ to $10^{-9} \mathrm{M}$ (Natarajan \& Dugdale, 1966; Carlucci \& Silbernagel, 1967). In some regions the concentration ranged below their limits of detection of about $10^{-13} \mathrm{M}$. This is still roo times the thiamine concentration of kinetic control for Cryptococcus albidus. Thus where thiamine is an important factor in the ecology of heterotrophic systems it is probably in a range below current means of analytical detection.

This investigation was supported by the Office of Naval Research Contract Nonr 30I0(05) and by the Office of Water Resources Research, U.S. Department of the Interior, project A-007-ALAS. Discussions with Dr R. C. Dugdale and the technical assistance of Miss E. R. Dieter are gratefully acknowledged.

Institute of Marine Science, Contribution No $5 \mathrm{I}$. 


\section{REFERENCES}

Amdur, I. \& Hammes, G. G. (1966). Chemical Kinetics: Principles and Selected Topics. New York: McGraw Hill.

BorkowskI, J. D. \& Johnson, M. J. (1967). Experimental evaluation of liquid film resistance in oxygen transport to microbial cells. Appl. Microbiol. 15, I483.

Butron, D. K. (1968). Selective thiamine removal from culture media by ultraviolet irradiation. Appl. Microbiol. 16, 530.

ButTon, D. K. ( $1969 a$ ). The effect of clay on the availability of dilute organic nutrients to steady state heterotrophic populations. Limonol. Oceanogr. (In the Press.)

Butron, D. K. (1969b). Some factors influencing kinetic constants for microbial growth in dilute solution. Proc. Symp. Organic Matter in Natural Waters. Ed by D. W. Hood. Institute of Marine Science, University of Alaska. (In the Press.)

Button, D. K. \& Garver, J. C. (I966). Continuous culture of Torulopis utilis: a kinetic study of oxygen limited growth. J. gen. Microbiol. 45, 195.

Carlucci, A. F. \& Silbernagel, S. B. (1967). Determination of vitamins in seawater. Proc. International Biology Program Symp., Amsterdam and Nieuwersluis.

Hengstenderg, W., Egan, J. B. \& Morse, M. L. (1967). Carbohydrate transport in Staphylococcus aureus. $\mathrm{V}$. The accumulation of phosphorylated carbohydrate derivatives, and evidence for a new enzyme-splitting lactose phosphate. Proc. natn. Acad. Sci. U.S.A. 58, 274.

Holm-Hansen, O., Strickland, J. D. H. \& Williams, P. M. (I966). A detailed analysis of biologically important substances in a profile off southern California. Limnol. Oceanogr. II, 548.

JANNASCH, H. W. (1967). Growth of marine bacteria at limiting concentrations of organic carbon in seawater. Limnol. Oceanogr. 12, 264.

Koser, S. A. (1968). Vitamin Requirements of Bacteria and Yeasts. Springfield, Ill.: Charles C. Thomas.

MülLER, R. (1967). Symposium über Hefe-Protoplasten. Abh. dt. Akad. Wiss. Berl.

Natarajan, K. V. \& Dugdale, R. C. (1966). Bioassay and distribution of thiamine in the sea. Limnol. Oceanogr. II, $62 \mathrm{I}$.

Neujahr, H. Y. (1966). Transport of B-vitamins in microorganisms II. Factors affecting the uptake of labelled thiamine by non-proliferating cells of Lactobacillus fermenti. Acta chem. scand. 2o, 771 .

Ziro, S. (1955). Thiamine uptake by yeast cells. J. Biochem. 42, 27. 